PROCEEDINGS OF THE

AMERICAN MATHEMATICAL SOCIETY

Volume 128, Number 8 , Pages 2451-2455

S 0002-9939(99)05456-8

Article electronically published on November 29, 1999

\title{
ADJACENCY PRESERVING MAPPINGS OF INVARIANT SUBSPACES OF A NULL SYSTEM
}

\author{
WEN-LING HUANG
}

(Communicated by Christopher Croke)

\begin{abstract}
In the space $I_{r}$ of invariant $r$-dimensional subspaces of a null system in $(2 r+1)$-dimensional projective space, W.L. Chow characterized the basic group of transformations as all the bijections $\varphi: I_{r} \rightarrow I_{r}$, for which both $\varphi$ and $\varphi^{-1}$ preserve adjacency. In the present paper we show that the two conditions $\varphi: I_{r} \rightarrow I_{r}$ is a surjection and $\varphi$ preserves adjacency are sufficient to characterize the basic group. At the end of this paper we give an application to Lie geometry.
\end{abstract}

\section{INTRODUCTION}

Let $n, r$ be positive integers, $3 \leq n=2 r+1$. Let $\Pi$ be an arbitrary $n$-dimensional Pappian projective space. A null system $\delta$ on $\Pi$ is a polarity on $\Pi$ which satisfies $x \in x^{\delta}$ for every point $x$ of $\Pi$. The space of the $r$-dimensional subspaces of $\Pi$ which are invariant under a fixed null system $\delta$ will be denoted by $I_{r}:=\left\{a \in[r] \mid a^{\delta}=a\right\}$, where $[k],-1 \leq k \leq n$, is the set of all $k$-dimensional subspaces of $\Pi$.

The basic group of transformations in the space $I_{r}$ (also called the group of semi-symplectic transformations) consists of the transformations induced by all the collineations $f$ of $\Pi$ which satisfy $\delta f=f \delta$. Two invariant $r$-dimensional subspaces $a, b$ are at distance $d$, if their intersection is $(r-d)$-dimensional. If $d=1$, then they are called adjacent.

W.L. Chow [4] has shown that any bijection $\varphi: I_{r} \rightarrow I_{r}$ for which both $\varphi$ and $\varphi^{-1}$ preserve adjacency is induced by a collineation of $\Pi$. Observably, any collineation $\varphi$ of $\Pi$ with $\delta \varphi=\varphi \delta$ preserves adjacency in both directions. From a different point of view, L.K. Hua [5], 6] proved the fundamental theorem in the geometry of symmetric matrices under further hypotheses. For a brief history of the development of this problem see Wan [9], [10]. We may consider the theorem of Chow as a Beckman-Quarles type theorem [8] on distance preserving mappings of the space $I_{r}$. Thus Chow's theorem may be seen as an early result in the discipline characterizations of geometrical mappings under mild hypotheses 3 .

In the present paper we characterize the basic group under mild hypotheses:

Theorem. Let $r, n \in \mathbb{N}, 3 \leq n=2 r+1$. Let $I_{r}$ be the space of all invariant $r$-dimensional subspaces of a null system $\delta$ in an $n$-dimensional Pappian projective

Received by the editors September 25, 1998.

1991 Mathematics Subject Classification. Primary 51A50; Secondary 51B25.

Key words and phrases. Null system, adjacency preserving mappings, symmetric matrices, Lie transformations. 
space $\Pi$. Let $\varphi: I_{r} \rightarrow I_{r}$ be a surjection satisfying

$$
\text { if } a, b \text { are adjacent, then } a^{\varphi}, b^{\varphi} \text { are adjacent }
$$

for all $a, b \in I_{r}$. Then $\varphi$ is a transformation of the basic group of $I_{r}$.

\section{Preliminaries}

In this paper, by dimension, intersection, and subspace we understand projective dimension, intersection, and subspace.

Let $n, r$ be integers, $3 \leq n=2 r+1$. Let $\Pi$ be an arbitrary $n$-dimensional Pappian projective space, and let $\delta$ be a null system on $\Pi$. For any subspaces $a, b$ of $\Pi$, we have the following well-known properties: $\operatorname{dim} a+\operatorname{dim} a^{\delta}=2 r,(a+b)^{\delta}=a^{\delta} \cap b^{\delta}$, $(a \cap b)^{\delta}=a^{\delta}+b^{\delta}$, and $a \subset b$ implies $b^{\delta} \subset a^{\delta}$. For $-1 \leq k \leq n$ let $[k]$ be the set of all $k$-dimensional subspaces of $\Pi$. For each $a \in[k]$, we call $\bar{a}:=a^{\delta} \in[2 r-k]$ the conjugate of $a$. An element $a \in[k]$ is called invariant, if $a \subset \bar{a}$ or $\bar{a} \subset a$. Let

$$
I_{k}:=\{a \in[k] \mid a \text { is invariant }\} .
$$

Two elements $a, b \in I_{r}$ are called adjacent if their intersection has dimension $r-1$. Let $a, b \in I_{r}$. The distance between $a$ and $b$ is defined to be $d(a, b):=r-\operatorname{dim}(a \cap b)$. If $a \neq b$, then $d=d(a, b)$ is the smallest positive integer with the property that there exists a sequence of $d+1$ invariant and consecutively adjacent subspaces $a_{1}=a, a_{2}, \ldots, a_{d+1}=b \in I_{r}$ (see [4]). From this property we obtain the triangle inequality $d(a, c) \leq d(a, b)+d(b, c)$ for all $a, b, c \in I_{r}$. Let $P \in I_{r-1}, a \in I_{r}$. We define the distance between $P$ and $a$ by

$$
d(P, a):=\min \left\{d(a, b) \mid b \in P^{*}\right\}=r-\operatorname{dim}(P \cap a)-1
$$

where $P^{*}:=\left\{l \in I_{r} \mid P \subset l\right\}$.

A subset $M$ of $I_{r}$ is called a maximal set of adjacent elements in $I_{r}$ if any two distinct elements of $M$ are adjacent and if there are no other elements of $I_{r}$ which are adjacent to each element of $M$.

Lemma 2.1. A set $M \subset I_{r}$ is maximal iff there is a $P \in I_{r-1}$ with $M=P^{*}$.

Proof. Assume there exists a "triangle" $a, b, c \in I_{r}$, such that $a, b$, resp. $b, c$, resp. $c, a$, are adjacent and $P:=a \cap b \neq b \cap c=: Q$. Then $H:=a+b=b+c=$ $c+a \in[r+1]$. Since $P \subset a$, we have $a=a^{\delta} \subset P^{\delta} \in I_{r+1}$ and $H=a+b=P^{\delta}$. Similarly, $H=b+c=Q^{\delta}$, i.e. $P^{\delta}=Q^{\delta}$ in contradiction to that $\delta$ is a one-to-one correspondence.

Let $\varphi: I_{r} \rightarrow I_{r}$ be a mapping which satisfies (1.1). Then $\left(P^{*}\right)^{\varphi}$ is contained in a unique maximal set $Q^{*}$. We define

$$
P^{\varphi}:=Q \quad \text { if } \quad\left(P^{*}\right)^{\varphi} \subset Q^{*} .
$$

Furthermore, following the definition of distance, for any $a, b \in I_{r}$ we have

$$
d(a, b) \geq d\left(a^{\varphi}, b^{\varphi}\right) .
$$

Lemma 2.2. For any invariant subspaces $x \in I_{s}, y \in I_{t}, t, s \leq r$, the subspace $x+(\bar{x} \cap y)$ is invariant and has dimension $\leq r$. In the case $r=t, x+(\bar{x} \cap y)$ has dimension $r$.

Lemma 2.3. For any $a \in I_{r}$ there exists $b \in I_{r}$ with $a \cap b=\emptyset$, i.e. $d(a, b)=r+1$.

For a proof of Lemma 2.2 and Lemma 2.3 ] see [4]. 
Lemma 2.4. For any $a, b \in I_{r}, a \neq b$, there exists $c \in I_{r}$ with $d(a, c)=r+1$ and $d(b, c)<r+1$.

Proof. Let $l \in I_{r}$ with $a \cap l=\emptyset$. If $b \cap l \neq \emptyset$, then take $c:=l$. Suppose $l$ satisfies $l \cap b=\emptyset$. Let $k:=\operatorname{dim}(a \cap b)<r$. Let $s$ be an $(r-k-1)$-dimensional subspace of $b$ with $s+(a \cap b)=b$. Following Lemma 2.2 $c:=s+(\bar{s} \cap l)$ is an invariant element with dimension $r$. It is clear that $b \cap c=s$ and $a \cap b \cap c=\emptyset$. Suppose $d(a, c) \neq r+1$. There is an $x \in a \cap c$. Hence $x \in c \subset \bar{s}$, and $s \subset \bar{x}$. On the other hand, $x \in a \subset \bar{x}$ implies $a \cap b \subset \bar{x}, b=(a \cap b)+s \subset \bar{x}$ and $x \in b$, a contradiction to $a \cap b \cap c=\emptyset$.

Lemma 2.5. Let $P \in I_{r-1}, a \in I_{r}$. Then $d(P, a)=k$ iff there is a uniquely determined $b \in P^{*}$ with $d(a, b)=k$ and $d(a, l)=k+1$ for any $l \in P^{*} \backslash\{b\}$.

Proof. " $\Rightarrow$ ": Let $b:=P+(a \cap \bar{P}) \in I_{r}$. Then $a \cap b=a \cap \bar{P}=\overline{a+P}$, hence $\operatorname{dim}(a \cap b)=2 r-\operatorname{dim}(a+P)=r-k$ and $d(a, b)=k$. For any $l \in P^{*} \backslash\{b\}$ we have $k \leq d(a, l) \leq d(a, b)+d(b, l)=k+1$. Let $l \in P^{*}$ with $d(a, l)=k$, i.e. $\operatorname{dim}(a \cap l)=r-k$. Since $a \cap l \subset a \cap \bar{P}$ and $\operatorname{dim}(a \cap l)=\operatorname{dim}(a \cap \bar{P}), a \cap \bar{P}=a \cap l \subset l$. Hence $l=P+(a \cap \bar{P})=b . \quad$ " $\Leftarrow$ " follows straightforwardly from the definition.

Lemma 2.6. Let $a, b \in I_{r}$ with $d(a, b)=k$. Then

$$
\min \left\{d(P, a) \mid P \subset b, P \in I_{r-1}\right\}=k-1 .
$$

Furthermore, for all $P \subset b, P \in I_{r-1}$ we have $d(P, a)=k-1$ iff $a \cap b \subset P$.

Proof. From Lemma 2.5, for all $P \subset b, P \in I_{r-1}$,

$$
k=d(a, b) \in\{d(P, a), d(P, a)+1\}=\{r-1-\operatorname{dim}(P \cap a), r-\operatorname{dim}(P \cap a)\} .
$$

Thus $a \cap b \subset P$ is equivalent to $\operatorname{dim}(P \cap a)=\operatorname{dim}(a \cap b)=r-k$ and to $d(P, a)=$ $k-1$.

Lemma 2.7. For any $a \in I_{r}$ and any projective point $x \in a$, there exists $b \in I_{r}$ with $d(a, b)=r$ and $a \cap b=\{x\}$.

Proof. Let $l \in I_{r}, l \cap a=\emptyset$. Define $b:=x+(\bar{x} \cap l) \in I_{r}$.

Lemma 2.8. Let $P \in I_{r-1}, a \in I_{r}$. Then for any mapping $\varphi: I_{r} \rightarrow I_{r}$ satisfying (1.1) we have $d(P, a) \geq d\left(P^{\varphi}, a^{\varphi}\right)$ where $P^{\varphi}$ is defined in (2.1).

Proof. Let $b \in P^{*}$ with $d(a, b)=d(P, a)$. Then $b^{\varphi} \in\left(P^{\varphi}\right)^{*}$ and

$$
d(P, a)=d(a, b) \geq d\left(a^{\varphi}, b^{\varphi}\right) \geq d\left(P^{\varphi}, a^{\varphi}\right) .
$$

\section{Proof of the theorem}

1. If there are $a, b \in I_{r}$ with $d(a, b)=r+1$ and $d\left(a^{\varphi}, b^{\varphi}\right)=r$, then for any $P \in I_{r-1}, P \subset a$ we have $a^{\varphi} \cap b^{\varphi} \subset P^{\varphi}$.

Proof. For any $P \in I_{r-1}, P \subset a$ we have $P^{\varphi} \subset a^{\varphi}$. Let $c \in P^{*} \backslash\{a\}$ with $d(b, c)=r$. Then $d\left(b^{\varphi}, c^{\varphi}\right) \leq r$ and $c^{\varphi} \in\left(P^{\varphi}\right)^{*}$. Since $a, c$ are adjacent, also $a^{\varphi}, c^{\varphi}$ are adjacent, and we have $c^{\varphi} \neq a^{\varphi}$. From Lemma 2.5 $d\left(P^{\varphi}, b^{\varphi}\right)=r-1$ and $a^{\varphi} \cap b^{\varphi} \subset P^{\varphi}$.

2. For any $a, b \in I_{r}, d\left(a^{\varphi}, b^{\varphi}\right)=r$ implies $d(a, b)=r$. 
Proof. Suppose not; then $d(a, b)=r+1$. Choose $c_{1}, \ldots, c_{r} \in I_{r}$ with $d\left(c_{i}^{\varphi}, a^{\varphi}\right)=r$ and such that the set $\left\{a^{\varphi} \cap c_{i}^{\varphi} \mid i=1, \ldots, r\right\} \cup\left\{a^{\varphi} \cap b^{\varphi}\right\}$ is a projective basis of $a^{\varphi}$. Then $r \leq d\left(c_{i}, a\right) \leq r+1$ for all $i=1, \ldots, r$. Choose $Q \subset a, Q \in I_{r-1}$ with $Q \cap c_{i}=a \cap c_{i}$ for all $i=1, \ldots, r$. In the case $d\left(a, c_{i}\right)=r+1$, from $1, a^{\varphi} \cap c_{i}^{\varphi} \subset Q^{\varphi}$. In the other case $d\left(a, c_{i}\right)=r$ we have $d\left(Q^{\varphi}, c_{i}^{\varphi}\right) \leq d\left(Q, c_{i}\right)=r-1$, so $a^{\varphi} \cap c_{i}^{\varphi} \subset Q^{\varphi}$ for all $i=1, \ldots, r$. Furthermore, $a^{\varphi} \cap b^{\varphi} \subset Q^{\varphi}$. This is a contradiction to that $\left\{a^{\varphi} \cap c_{i}^{\varphi} \mid i=1, \ldots, r\right\} \cup\left\{a^{\varphi} \cap b^{\varphi}\right\}$ is a basis of $a^{\varphi}$.

3. For any $a \in I_{r}, Q \in I_{r-1}, Q \subset a^{\varphi}$, there exists $P \in I_{r-1}, P \subset a$ with $P^{\varphi}=Q$.

Proof. Choose $c_{1}, \ldots, c_{r} \in I_{r}$ with $d\left(a^{\varphi}, c_{i}^{\varphi}\right)=r$ such that $\left\{a^{\varphi} \cap c_{i}^{\varphi} \mid i=1, \ldots, r\right\}$ is a basis of $Q$. Then for any $P \in I_{r-1}$ with $P \subset a, a \cap c_{i} \subset P$ implies $a^{\varphi} \cap c_{i}^{\varphi} \subset P^{\varphi}$ and $P^{\varphi}=Q$.

4. For any $a, b \in I_{r}$ with $d(a, b)=r+1$, we have $d\left(a^{\varphi}, b^{\varphi}\right)=r+1$.

Proof. We prove 4 by induction. From 2, $d\left(a^{\varphi}, b^{\varphi}\right) \neq r$. Let $d\left(a^{\varphi}, b^{\varphi}\right) \neq r+1-k$ for some $k \in\{1, \ldots, r\}$. Assume that $d\left(a^{\varphi}, b^{\varphi}\right)=r-k$. Let $Q \subset a^{\varphi}$ with $d\left(Q, b^{\varphi}\right)=r-k$. Let $P \subset a$ with $P^{\varphi}=Q$. Choose $l \in P^{*} \backslash\{a\}$ with $d(l, b)=r+1$. Then, by Lemma 2.5, $l^{\varphi} \in Q^{*} \backslash\left\{a^{\varphi}\right\}$ implies $d\left(l^{\varphi}, b^{\varphi}\right)=r+1-k$, a contradiction. Hence $d\left(a^{\varphi}, b^{\varphi}\right) \neq r-k$.

5. $\varphi$ is injective.

Proof. For any $a \neq b \in I_{r}$, from Lemma 2.4 there exists $c \in I_{r}$ with $d(a, c)=r+1$ and $d(b, c)<r+1$. Since $d\left(a^{\varphi}, c^{\varphi}\right)=r+1$ and $d\left(b^{\varphi}, c^{\varphi}\right) \leq d(b, c)<r+1$, we have $a^{\varphi} \neq b^{\varphi}$.

6. $a, b \in I_{r}$ are adjacent if $a^{\varphi}, b^{\varphi}$ are adjacent.

Proof. Choose $c \in I_{r}$ with $d\left(a^{\varphi}, c^{\varphi}\right)=r+1$ and $d\left(b^{\varphi}, c^{\varphi}\right)=r$. Denote $Q:=a^{\varphi} \cap b^{\varphi}$. Let $P \subset a, P \in I_{r-1}$ with $P^{\varphi}=Q$. Let $l \in P^{*}$ with $d(l, c)=r$; then $l \neq a$. Since $l^{\varphi} \in Q^{*}, r=d\left(Q, c^{\varphi}\right) \leq d\left(l^{\varphi}, c^{\varphi}\right) \leq d(l, c)=r$. Following Lemma 2.5, we have $l^{\varphi}=b^{\varphi} . \varphi$ is injective, hence $l=b$. So $a$ and $b$ are adjacent.

7. $\varphi$ is a transformation of the basic group of $I_{r}$.

Proof. From [5, and 6., $\varphi$ is a bijection of $I_{r}$, and $\varphi$ and $\varphi^{-1}$ preserve adjacency of pairs of elements of $I_{r}$. Chow's theorem completes the proof of the theorem.

\section{Applichtion to lie geometry}

Let $\mathbf{Q}$ denote the Lie quadric

$$
x_{1} x_{2}+x_{3} x_{4}+x_{5}^{2}=0
$$

in the four-dimensional projective space $\Pi^{4}(K)$ over a commutative field $K, \operatorname{ch} K \neq$ 2. We call two elements $X=K\left(x_{1}, \ldots, x_{5}\right), Y=K\left(y_{1}, \ldots, y_{5}\right)$ of $\mathbf{Q}$ conjugate if

$$
X \sim Y \quad: \Leftrightarrow \quad x_{1} y_{2}+x_{2} y_{1}+x_{3} y_{4}+x_{4} y_{3}+2 x_{5} y_{5}=0 .
$$

The Lie transformations of $(\mathbf{Q}, \sim)$ are defined as bijections $\alpha$ of $\mathbf{Q}$ satisfying $X \sim Y$ iff $X^{\alpha} \sim Y^{\alpha}$. Every Lie transformation is induced by a collineation of $\Pi^{4}(K)$ (see e.g. [2]).

On the three-dimensional projective space $\Pi^{3}(K)$ we define a null system $\delta$ by

$$
P^{\delta}=\left\{Q \mid p_{1} q_{3}-p_{3} q_{1}+p_{2} q_{4}-p_{4} q_{2}=0\right\}
$$


where $P=K\left(p_{1}, \ldots, p_{4}\right), Q=K\left(q_{1}, \ldots, q_{4}\right)$. There is a one-to-one correspondence $\gamma$ between the space of invariant lines $I_{1}$ and the Lie quadric $\mathbf{Q}$ which satisfies

$$
a, b \text { are adjacent } \Leftrightarrow a^{\gamma}, b^{\gamma} \text { are distinct and conjugate }
$$

for all $a, b \in I_{1}$. This transformation $\gamma$ is defined as follows. For every line $a$ of $\Pi^{3}(K)$ consider the Plücker coordinates $K\left(a_{12}, a_{13}, a_{14}, a_{34}, a_{42}, a_{23}\right)$ of $a$ where $a_{i j}=p_{i} q_{j}-p_{j} q_{i}$ for any two distinct points $P, Q \in a, P=K\left(p_{1}, \ldots, p_{4}\right), Q=$ $K\left(q_{1}, \ldots, q_{4}\right)$. Then $a_{13}=a_{42}$ if, and only if, $a=a^{\delta}$, i.e. $a \in I_{1}$. Define $\gamma: I_{1} \rightarrow$ $\mathbf{Q}$ by $a \mapsto K\left(a_{12}, a_{34}, a_{14}, a_{23}, a_{13}\right)$. $\gamma$ is a bijection. Furthermore, any distinct $a, b \in I_{1}$ with Plücker coordinates $K\left(a_{12}, a_{34}, a_{14}, a_{23}, a_{13}\right), K\left(b_{12}, b_{34}, b_{14}, b_{23}, b_{13}\right)$ are adjacent if, and only if,

$$
\begin{aligned}
& a_{12} b_{34}+a_{34} b_{12}+a_{13} b_{42}+a_{42} b_{13}+a_{14} b_{23}+a_{23} b_{14}=0 \\
\Leftrightarrow & a_{12} b_{34}+a_{34} b_{12}+a_{14} b_{23}+a_{23} b_{14}+2 a_{13} b_{13}=0 \\
\Leftrightarrow & a^{\gamma}, b^{\gamma} \text { are conjugate. }
\end{aligned}
$$

In the case $(r, n)=(1,3)$, the theorem implies the following corollary.

Corollary 4.1. Let $\psi: \mathbf{Q} \rightarrow \mathbf{Q}$ be a surjective mapping which takes pairs of distinct conjugate points of $\mathbf{Q}$ to pairs of distinct conjugate points. Then $\psi$ is a Lie transformation.

\section{REFERENCES}

[1] R. Baer. Linear Algebra and Projective Geometry. Academic Press, New York, San Francisco, London, 1952. MR 14:675j

[2] W. Benz. Geometrie der Algebren. Springer-Verlag, Berlin Heidelberg New York, 1973. MR 50:5623

[3] W. Benz. Geometrische Transformationen. BI Wissenschaftsverlag, Mannheim; Leipzig; Wien; Zürich, 1992. MR 93i:51002

[4] W.-L. Chow. On the geometry of algebraic homogeneous spaces. Ann. Math., 50(1):32-67, 1949. MR 10:396d

[5] L.K. Hua. Geometries of matrices III. Fundamental theorems in the geometries of symmetric matrices. Trans. Amer. Math. Soc., 61:229-255, 1947. MR 9:171e

[6] L.K. Hua. Geometries of symmetric matrices over any field with characteristic other than two. Ann. Math., 50:8-31, 1949. MR 10:424h

[7] W.-l. Huang. Adjacency preserving mappings of Grassmann spaces. Abh. Math. Sem. Univ. Hamburg, 68:65-77, 1998. CMP 99:05

[8] J. A. Lester. Distance preserving transformations. In F. Buekenhout, editor, Handbook of Incidence Geometry, pages 921-944, Amsterdam, 1995. Elsevier. MR 96j:51019

[9] Z.-X. Wan. Geometry of matrices. Adv. Stud. Pure Math., 24:443-453, 1996. MR 97h:15017

[10] Z.-X. Wan. Geometry of matrices. World Scientific, Singapore, 1996. MR 98a:51001]

Mathematisches Seminar, Universität Hamburg, Bundesstrasse 55, 20146 Hamburg, GERMAnY

E-mail address: huang@math.uni-hamburg.de 